\title{
Velocity Component Comparison between CFD and EFD in Taylor Vortex Flow
}

\author{
Hiroyuki Furukawa and Noritaka Suzuki \\ Department of Mechanical Engineering, Meijo University, Nagoya 468-8502, Japan
}

\begin{abstract}
Since the classical investigation of the Taylor vortex by G. I. Taylor in 1923, many researchers have studied the Taylor vortex as one of the most important vortex types in flow. In this study, the inner cylinder is rotating, while the outer cylinder, which is concentric with the inner cylinder, is stationary. In addition, the measurement of the velocity distribution is carried out by the PIV (Particle Image Velocimetry) method. The radius of the inner cylinder is $20 \mathrm{~mm}$, and that of the outer cylinder is $30 \mathrm{~mm}$. In this study, $R e=650-1,200$ is assumed. In the upper part of the apparatus, movable ends are fixed to the upper and lower sides of the cylinder to change the aspect ratio. The aspect ratio is defined as the ratio of cylinder height to gap distance. A servo motor to rotate the inner cylinder, a servo-motor control device, a servo amplifier for rotation speed control, and a YAG laser light source are installed in the apparatus. For the visualization of Taylor vortex flow, aluminum powder composed of scale like fine particles is used. As tracer particles used in the PIV method, fluorescent particles with a size of $48 \mu \mathrm{m}$ were used. The governing equations are Navier-Stokes equations with cylindrical coordinates $(r, \theta, z)$ and the equations of continuity. Each physical value is nondimensionalized using the angular velocity of the inner cylinder as the representative velocity, and the radius difference between the inner and outer cylinders as the representative length. Discretization of the governing equations is based on the MAC method. The results of EFD and CFD (computational fluid dynamics) are compared. The mode bifurcation is observed, and the flow structure is investigated.
\end{abstract}

Key words: Taylor vortex flow, PIV, bifurcation, time dependent flow.

\section{Introduction}

Taylor vortex flow has been studied as an important vortex flow since it was first reported by G. I. Taylor in 1923 [1]. In a concentric double cylinder, when the rotation speed of the inner cylinder is gradually increased from zero, Couette flow first occurs in the gap between the inner and outer cylinders. When the rotation speed of the inner cylinder is further increased, Couette flow changes to Taylor vortex flow, in which many torus flows called cells are stacked, then to wavy Taylor vortex flow, and finally to turbulent flow $[2,3]$. Taylor vortex flow often appears in journal bearings, hydrodynamic machines, and containers for chemical reactions, and clarification of the mechanism of Taylor vortex flow is highly important for the engineering field. Especially, when the cylinder length

Corresponding author: Hiroyuki Furukawa, Ph.D., associate professor, research field: fluid dynamics. is small, unique vortex flow appear [4-7]. As the inner cylinder speed is changed, the flow bifurcation occurs, and it affects rapid change of the physical parameters such as torque on the cylinder-wall [8-10]. Though numerical studies show the flow structure of Taylor vortex flow [11-15], a few experimental investigations analyze the velocity vector field in Taylor vortex flow. Fig. 1 shows a schematic of Taylor vortex flow.

The main parameters that govern Taylor vortex flow generated in concentric double cylinders of finite length are the aspect ratio, which is the ratio of the cylinder height to the width of the gap between the inner and outer cylinders, and the Reynolds number, which depends on the rotation speed of the inner cylinder. Various types of Taylor vortex flow are formed depending on these parameters. The structure of Taylor vortex flow is roughly classified into two modes: normal mode and anomalous mode. In the case of a concentric double cylinder with the upper 


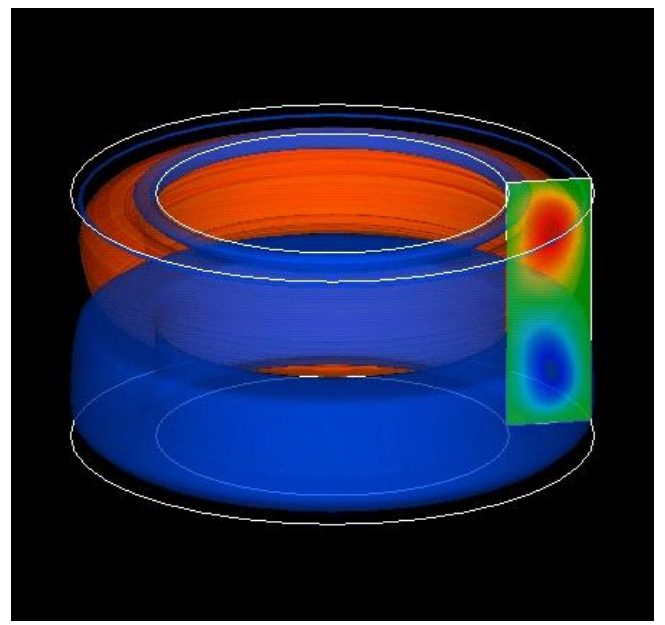

Fig. 1 Taylor vortex flow.

and lower ends fixed, the normal mode exhibits inward flows from the outer cylinder to the inner cylinder at both the upper and lower ends. In contrast, the anomalous mode exhibits outward flows at one or both ends of the cylinders. The purpose of this study is to analyze the transition processes of unsteady Taylor vortex flow by PIV (Particle Image Velocimetry). Moreover, the size of extra vortices generated during the transition process is measured and the anomalous one-cell mode is also analyzed by PIV.

\section{Experimental Setup and Procedure}

Fig. 2 shows a schematic of the experimental setup used in this study. The radii of the inner and outer cylinders are $r_{\text {in }}=20 \mathrm{~mm}$ and $r_{\text {out }}=30 \mathrm{~mm}$, respectively, and the gap between the cylinders is $r_{\text {out }}$ $-r_{\text {in }}=10 \mathrm{~mm}$. The inner cylinder is rotated at an angular velocity of $\omega$. A pulley is attached to a belt wheel below the center of the cylinder to rotate the inner cylinder. Here, only the inner cylinder is rotated because the outer cylinder is fixed. A slide ring is attached to the upper ends of the cylinders to fix their positions and to change the aspect ratio of the gap, which is used as the observation target. The setup also includes a servo motor, a servo amplifier (MR-J2-A, Mitsubishi Electric Corporation), a servo motor control unit (Tateiwa Shoji), and a Nd-YAG laser device (1.5 W, DSPP Green Laser, Japan Laser Corporation).

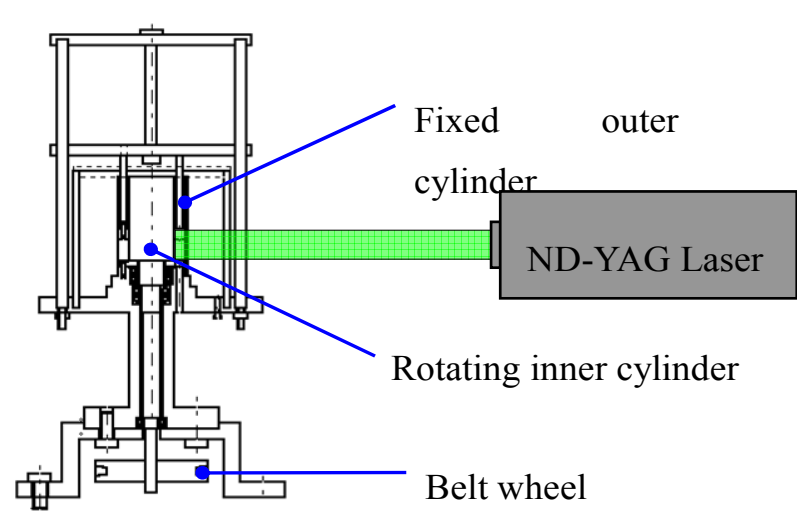

Fig. 2 Schematic of experimental setup.

A mixture of distilled water and glycerin with a mixing ratio of $8: 2$ is used as the fluid specimen in this study. The specific gravity of glycerin is 1.26. The advantages of using glycerin are that Eq. (1) the Reynolds number can be controlled by varying its viscosity and Eq. (2) it helps tracer particles float in the fluid specimen. The tracer particles used in this study are fine fluorescent particles with a high luminescence, which were added to the fluid to visualize flows. FLUOSTAR (EBM Corporation) particles are used in this study. When laser light is irradiated onto these particles perpendicularly to the observation direction, flows can be observed owing to the reflection of light.

The aspect ratio was determined, and the rotation speed of the servo motor required to give the desired Reynolds number and the time required to reach this value (i.e., the rising time) were input to the servo amplifier. Next, the motor was driven and photographs were taken using a high-speed video camera (VCC-H1000, Digimo Co., Ltd.) when the vortex stabilized to form the final-mode flow. The obtained video data were sent to a PC and converted into a file of successive images, which were analyzed using two types of analytical software Koncerto (Seika Corporation). The parameters that govern Taylor vortex flows are the Reynolds number and aspect ratio, as mentioned above. The Reynolds number $R e$ is a dimensionless number, defined as the ratio of the inertial force in the equation of motion to the viscous force, and is given by 


$$
R e=\frac{V d}{v}
$$

here, $V$ is a representative velocity, given as the rotation speed of the inner cylinder $\left(r_{\mathrm{in}}, \omega\right), d$ is the width of the gap between the inner and outer cylinders, given by the difference between their representative radii as $r_{\text {out }}-r_{\text {in }}$, and $v$ is the kinematic viscosity of the fluid. The aspect ratio $\Gamma$ is defined as the ratio of the cylinder length $L$ to the gap width $r_{\text {out }}-r_{\text {in }}$, and is given by

$$
\Gamma=\frac{L}{r_{\text {out }}-r_{\text {in }}}
$$

Various final modes of Taylor vortex flow are formed by changing these parameters.

\section{PIV Analysis}

PIV is a method used to quantify flows to enable their visualization. Fig. 3 shows a schematic of the analytical method. A video of flows visualized using tracer particles is recorded using a high-speed video camera. The video sequences are segmented into frames of still images. The left and center images in Fig. 3 represent two successive extracted still images. Observing the movement of particles from time $t_{1}$ to $t_{2}$ reveals the velocity components in each still image, from which velocity vectors can be obtained.

\section{Experimental Results}

Transition from four-cell mode to two-cell mode in this study, using PIV we analyzed the transition from the normal four-cell (N4) mode to the normal two-cell (N2) mode when $\Gamma$ was 3.0 as well as the transition from the normal six-cell (N6) mode to the N4 mode when $\Gamma$ was 5.0. The results of PIV analysis for the former transition, obtained by recording at $250 \mathrm{fps}$, are described in this section. Fig. 4 shows the change in $R e$ with time during the transition. Re was decreased from 1,000 to 300 in 5 s. Fig. 5 shows the results of PIV analysis for the flow during the transition. Recording was started $1 \mathrm{~s}$ after the rotation speed of the inner cylinder was changed $(t=1)$. In Fig. 5, warm-colored arrows represent high velocities, whereas cold-colored arrows represent low velocities. The images in Fig. 5 revealed that the transition of the vortices started at $t=1.544$, and the three-cell mode was formed at $t=1.744$. The flow underwent a transition to the two-cell mode at $t=3.064$ and then to the $\mathrm{N} 2$ mode at $t=4.4$. The transition of the vortices is explained as follows. First, the second vortex from the top disappeared to form the three-cell mode. Next, the central vortex absorbed the top vortex, which rotated in the same direction as the central vortex, to form the two-cell mode. Finally, the bottom vortex developed to form the N2 mode. Measurement of size of extra vortex generated during transition. The size of the extra vortex that appeared at the bottom-left corner of A1 when $R e$ was 1,000 and $\Gamma$ was 0.9 was measured. Compared with the conventional method, enlarged images can be obtained by combining a macro zoom lens and FLUOSTAR particles. The extra vortex was observed in $3 \times 3 \mathrm{~mm}^{2}$ images, and the distances from the red dot to the red bars were measured, as shown in Fig. 6. Graphs of the radial and axial velocities of the extra vortex were not prepared. In 2011, Tanaka et al. estimated the size of the extra vortex to be approximately $2 \mathrm{~mm}$ from its images.
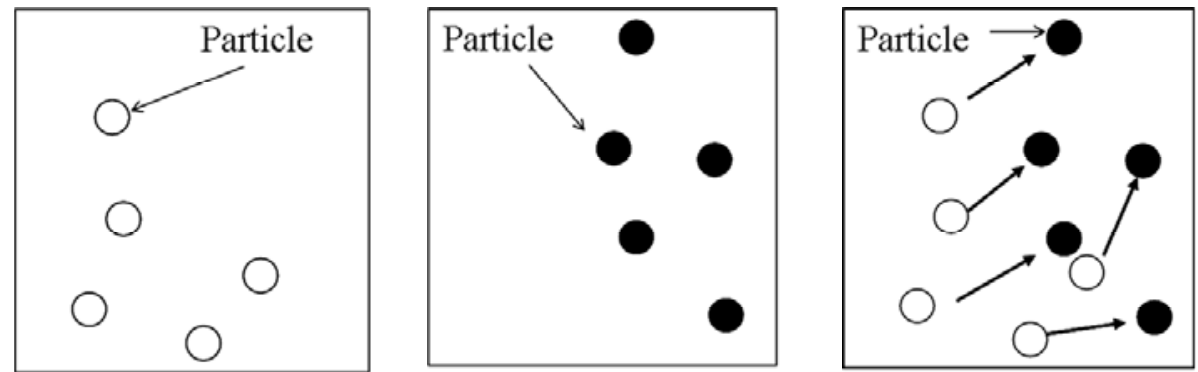

Fig. 3 PIV analytical method. 


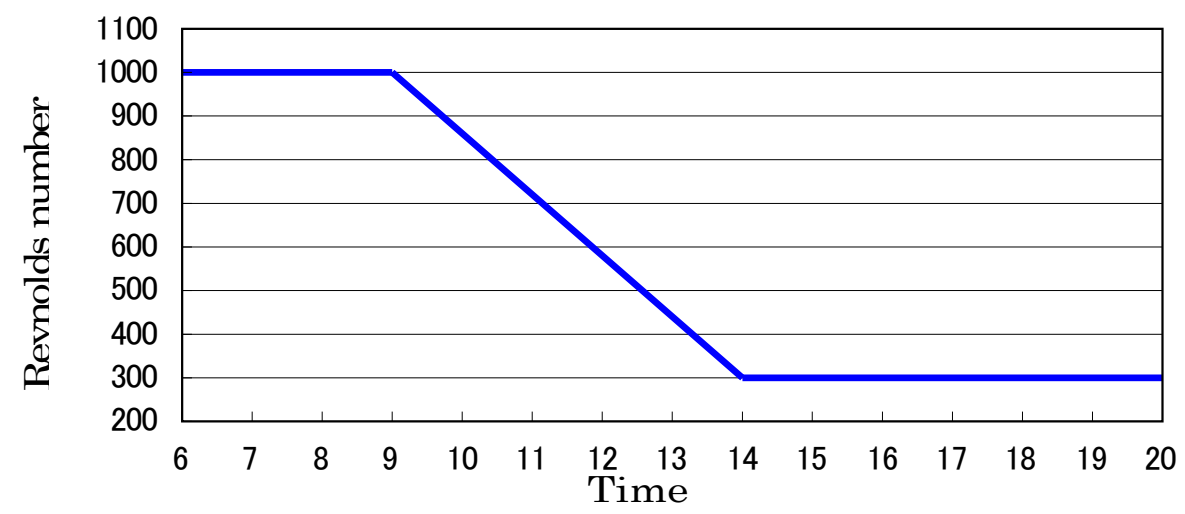

Fig. 4 How to change in $R e$.

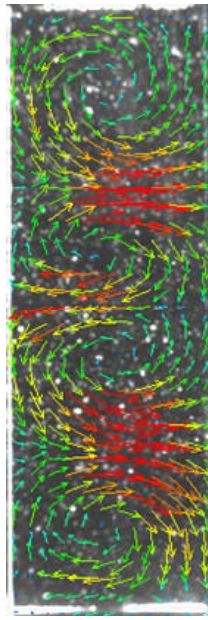

$t=1.00$

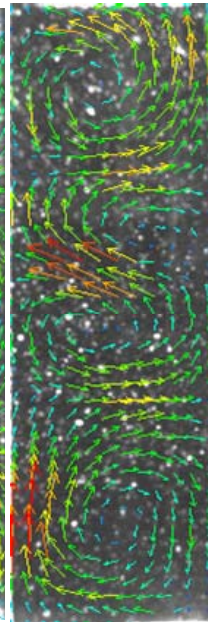

1.544

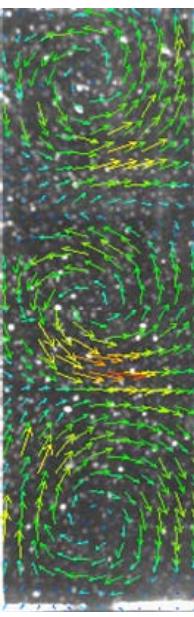

1.744

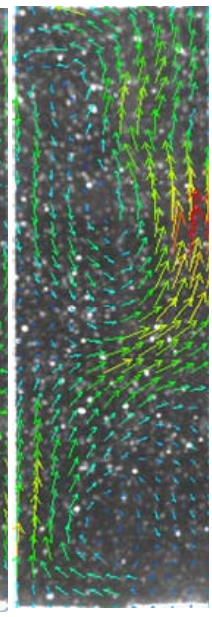

2.480

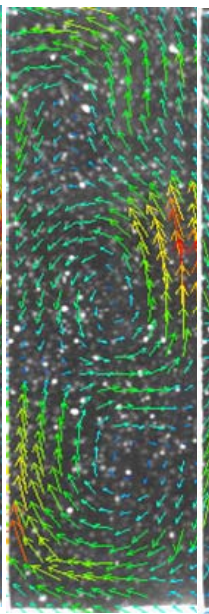

3.064

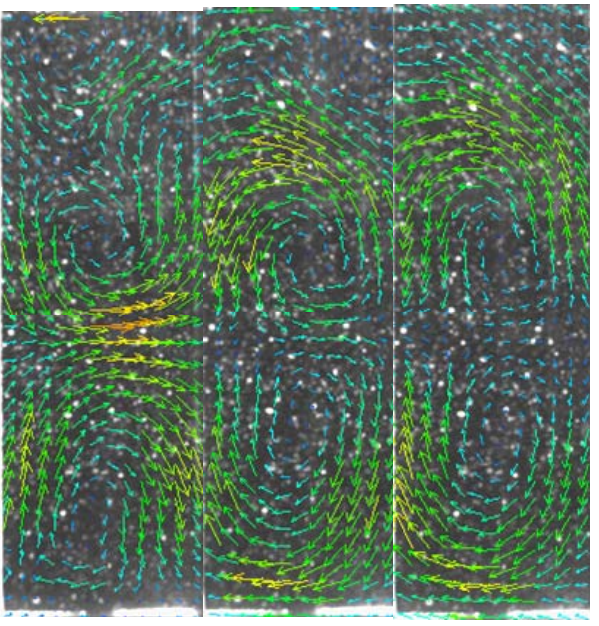

3.986

4.080

4.400

Fig. 5 PIV results $(R e=1,000 \rightarrow 300, \Gamma=3.0,250 \mathrm{fps})$.
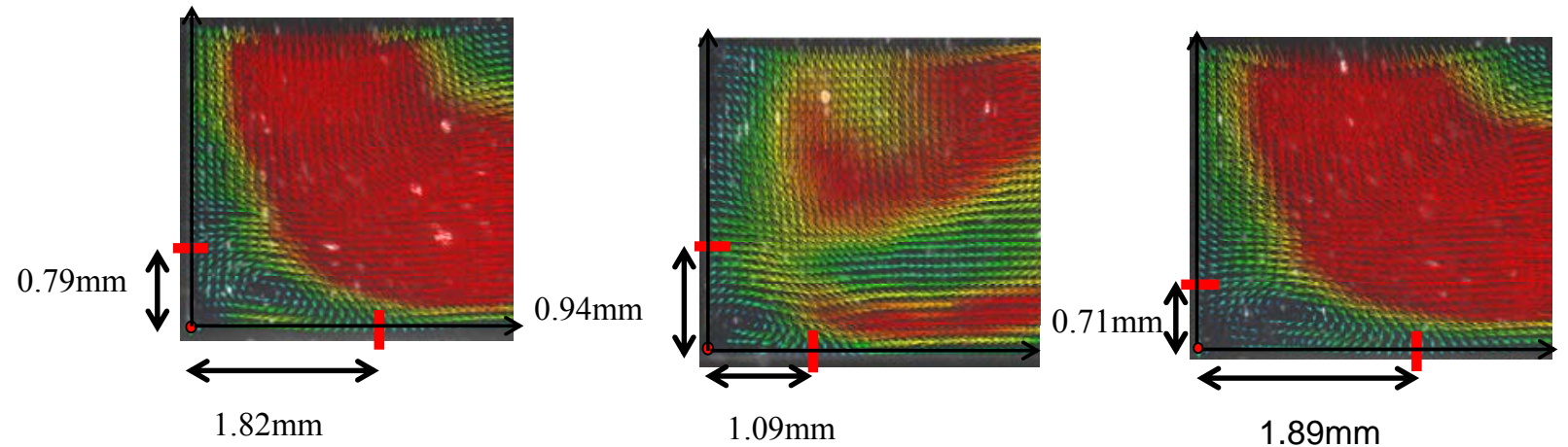

Fig. 6 PIV results for extra vortex $(\operatorname{Re}=1,000, \Gamma=0.9,250 \mathrm{fps})$.

Actual measurement in this study revealed that the axial and radial diameters were in the ranges of $0.7-1.02 \mathrm{~mm}$ and $1.09-1.89 \mathrm{~mm}$, respectively. That is, the smallest extra vortex had an axial diameter of 0.7 $\mathrm{mm}$ and a radial diameter of $1.09 \mathrm{~mm}$, whereas the largest extra vortex had an axial diameter of $1.02 \mathrm{~mm}$ and a radial diameter of $1.89 \mathrm{~mm}$. It was confirmed that the extra vortex that was generated had a height of approximately $10 \%$ of the cylinder length $(9 \mathrm{~mm})$ and a width of approximately $11-19 \%$ of the gap width (10 
$\mathrm{mm})$. Therefore, the diameter of the extra vortex was not considered to exceed $2 \mathrm{~mm}$.

The variation in the size of the extra vortex, as observed in this study, may be due to the effect of the neighboring large vortex that exists above the extra vortex. The radial and axial velocities at the center of the extra vortex will be obtained by furthering this study in the future.

\section{Improvement of Accuracy: N2 Mode}

Fig. 7 shows the results reported by Tanaka et al. in 2011. The values in the area enclosed by the red circle had large errors and were corrected as shown in Fig. 8. The area enclosed by the red circle, i.e., the area with high velocities, was enlarged to obtain the right-hand image in Fig. 8. The maximum dimensionless velocity was 0.238 at a dimensionless distance of approximately 0.7 according to the results of a CFD (computational fluid dynamics) simulation, whereas it was 0.1645 at approximately 0.8 according to the results of PIV analysis before correction. The difference between them was 0.0735 , giving an error of $30.9 \%$. When the result of PIV analysis was corrected, the maximum dimensionless velocity became 0.1978 at a dimensionless distance 1.0. The error was $16.9 \%$, which was $14 \%$ smaller than the error before correction. Therefore, enlarging the area with high velocities in the analysis can reduce the error and increase the accuracy of analysis.

\section{Improvement of Accuracy: N6 Mode}

The accuracy of analysis for the N6 mode was improved by enlarging two areas as shown in Fig. 9. The maximum dimensionless velocity in red circle 1 obtained by the CFD simulation was 0.281 , whereas it was 0.111 at a dimensionless distance of approximately 1.0 according to the results of PIV analysis before correction. The difference between these values was 0.17 , giving a large error of $60.5 \%$. After the correction of the PIV analytical result, the maximum dimensionless velocity became 0.219 at the same dimensionless distance of approximately 1.0, a difference of 0.062 from the value obtained by the CFD simulation. The error was $22.1 \%$, which was over $35 \%$ smaller than the error before correction. The minimum dimensionless velocity in red circle 2 obtained by the CFD simulation was -0.177 , whereas it was -0.12 according to the results of PIV analysis

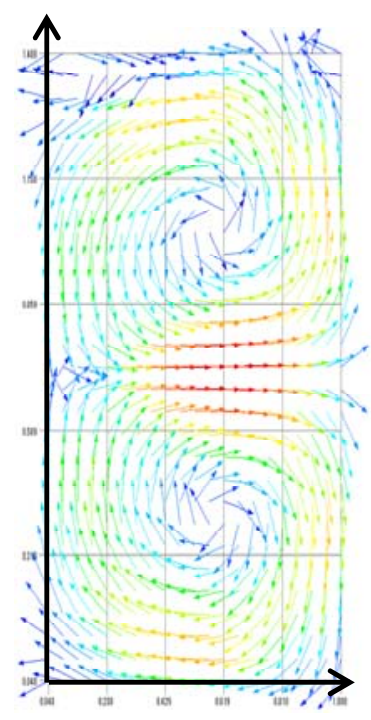

(1) Normalized PIV result

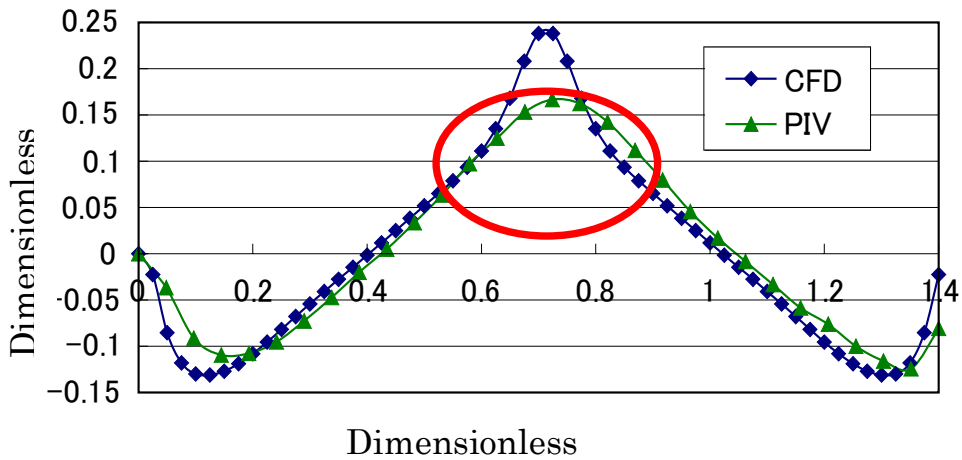

(2) Dimensionless velocity

Fig. 7 PIV results before improvement $(\operatorname{Re}=1,400, \Gamma=1.4,250 \mathrm{fps})$. 
before correction. The difference between these values was 0.057 , giving an error of $32.2 \%$. After the correction of the PIV analytical result, the minimum dimensionless velocity became -0.17 , a difference of 0.007 from the result of the CFD simulation. The error was $4 \%$, indicating that the PIV result after correction

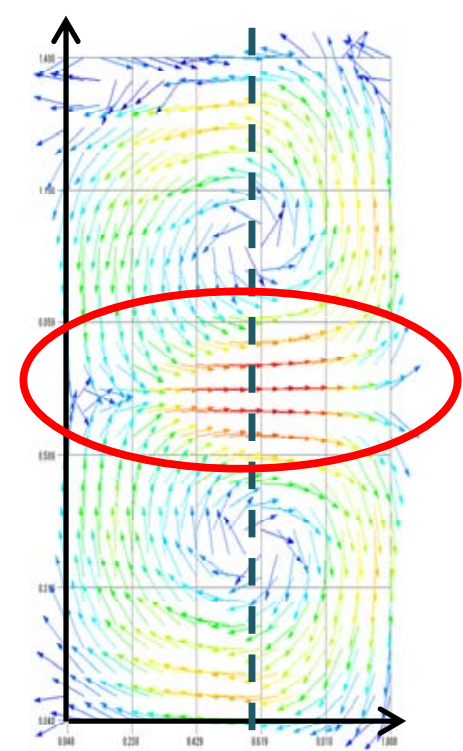

(1)Normalized PIV result was in good agreement with that obtained by the CFD simulation. From above, the accuracy for targets observed with a large aspect ratio can be markedly improved by first analyzing the flow throughout the target then analyzing enlarged images of the area between vortices.

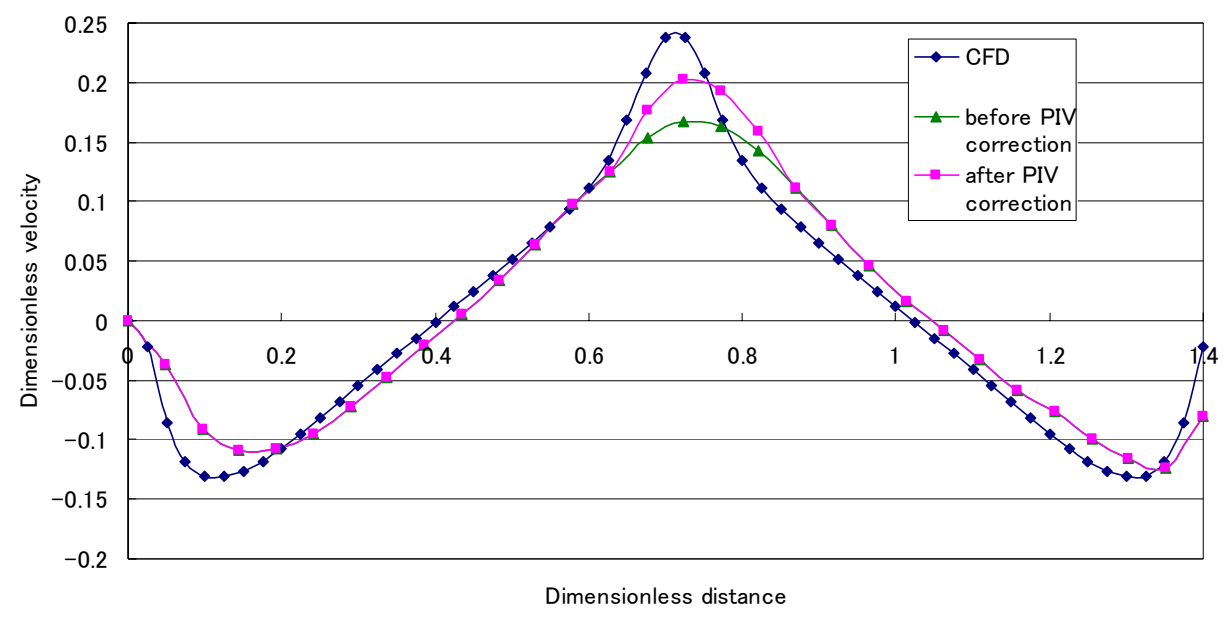

(3) Velocity distribution after correction

Fig. 8 Improvement of accuracy (N2). 

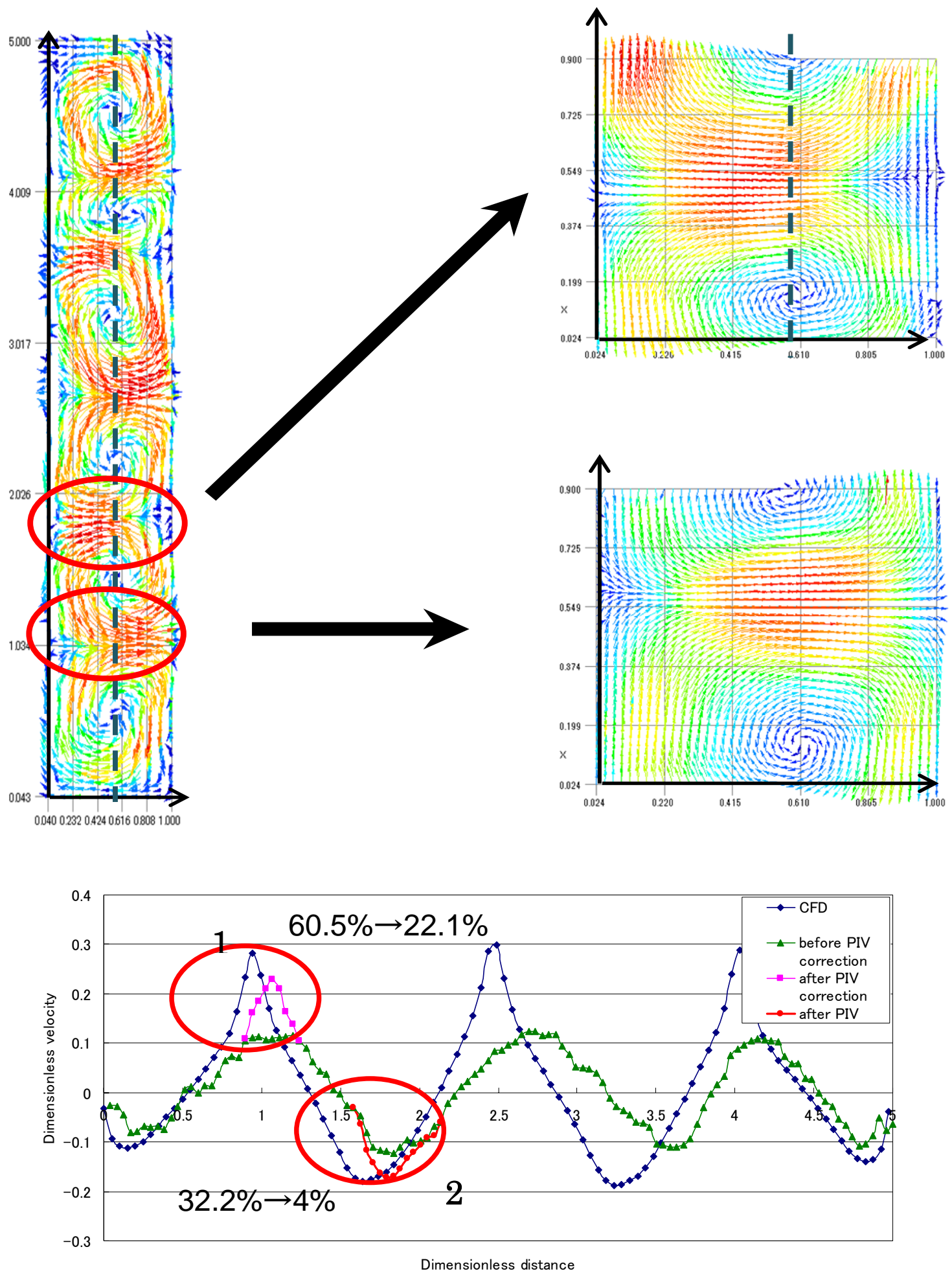

Fig. 9 Improvement of accuracy $(\mathrm{N6}, \operatorname{Re}=1,000, \Gamma=5.0,250 \mathrm{fps})$. 


\section{Conclusions}

Transition from four-cell mode to two-cell mode, we analyzed the transition from the $\mathrm{N} 4$ mode to the $\mathrm{N} 2$ mode and that from the N6 mode to the N4 mode and successfully analyzed the unsteady flows in detail. In the transition from the N6 mode to the N4 mode, the second vortex from the top disappeared and one of the vortices was absorbed by another vortex with the same rotation direction. The process of disappearance of the vortex in the transition from the N4 mode to the N2 mode was considered to be similar to that in the transition from the N6 mode to the N4 mode.

In the experiment, only the above-mentioned transitions were observed. However, different transition processes were also observed in the numerical simulation, which should have also been observed in the experiments. Although an abrupt change in the rotation speed of the inner cylinder was required to induce the transition of flows in this study, it was considered that transitions other than those mentioned above can also be experimentally observed by gradually changing the rotation speed of the inner cylinder.

\section{References}

[1] Taylor, G. I. 1923. "Stability of a Viscous Liquid Contained between Two Rotating Cylinders." Phil. Trans. Roy. Soc. Lond A 233: 289-343.

[2] Benjamin, T. B., and Mullin, T. 1982. "Notes on the Multiplicity of Flows in the Taylor Experiment." J. Fluid Mech. 121: 219-30.

[3] Tagg, R. 1994. "The Couette-Taylor Problem.” Nonlinear Science Today 4-3: 1-25.
[4] Benjamin, T. B., and Mullin, T. 1981. "Anomalous mode in the Taylor Experiment." In Proceedings of the Royal Society A, 221-49.

[5] Cliffe, K. A. 1983. "Numerical Calculation of Two-Cell and Single Cell Taylor Flow." J. Fluid Mech. 135: 219-33.

[6] Lücke, M., Mihelcic, M., Wingerath, K., and Pfister, G. 1984. "Flow in a Small Annulus between Concentric Cylinders." J. Fluid Mech. 140: 343-53.

[7] Bolstad, J. H., and Keller, H. B. 1987. "Computation of Anomalous Modes in the Taylor Experiment." J. Comput. Phys. 69: 230-51.

[8] Benjamin, T. B. 1979. "Bifurcation Phenomena in Steady Flows of a Viscous Fluid II. Experiment." In Proceedings of the Royal Society A, 27-43.

[9] Lücke, M., Mihelcic, M., and Wingerath, K. 1985. "Front Propagation and Pattern Formation of Taylor Vortices Growing into Unstable Circular Coquette Flow." Phy. Rev A 31-1: 396-409.

[10] Cliffe, K. A. 1988. "Numerical Calculation of the Primary Flow Exchange Process in the Taylor Problem." J. Fluid Mech. 197: 57-79.

[11] Alziary de Roquefort, T., and Grillaud, G. 1978. "Computation of Taylor Vortex Flow by a Transient Implicit Method." Comput. Fluids 6: 259-69.

[12] Neizal, G. P. 1984. "Numerical Computation of Time Dependent Taylor-Vortex Flows in Finite-Length Geometries." J. Fluid Mech. 141: 51-66.

[13] Cliffe, K. A., and Mullin, T. A. 1985. "Numerical and Eexperimental Study of Anomalous Modes in the Taylor Experiment." J. Fluid Mech. 153: 243-58.

[14] Liao, C. B., Jane, S. J., and Young, D. L. 1999. "Numerical Simulation of Three-Dimensional Couette-Taylor Flows." Int. J. Numer. Meths. Fluids 29: 827-47.

[15] Furukawa, H., Watanabe, T., and Nakamura, I. 2000. "Visual Information of the Mode Formation in Accelerating Taylor Vortex Flow with Finite Length." Proc. 9th Int. Symp. Flow Visual. 